\title{
Activation of Monocyte-Macrophage Cells in Pregnancies Complicated by Bacterial-Viral Urogenital Infections
}

\author{
L.A.Vygovskaya* \\ Candidate of Medical Science, Associate professor at the Department of Obstetrics and Gynecology and Children \\ Gynecology, Kharkov National Medical University, 4 Lenina avenue, Kharkov 61022.
}

Received: November 01, 2016; Accepted: November 08, 2016; Published: November 19, 2016

*Corresponding author: Liudmyla Anatolyevna Vygivska, Candidate of Medical Science, Associate professor at the Department of Obstetrics and Gynecology and Children Gynecology, Kharkov National Medical University, 4 Lenina avenue, Kharkov 61022, Tel: +380509675487;E-mail: liudmilavygovskaya@gmail.com.

\begin{abstract}
Introduction: Fetoplacental insufficiency and intrauterine infection of the fetus are one of the triggers for various complications of gestational process with persistent infections of the pregnant being a special concern for obstetricians. Immunopathogenic mechanisms play the leading role in the development of these complications. Pregnancy is known to be characterized by a unique new equilibrium state between specific and nonspecific immunity in the mother in which monocytes, rather than lymphocytes become the central cells of immunological adaptation.
\end{abstract}

\section{The purpose of the study}

Is to assess the changes in expression of the functional molecules on the surface of monocytes in the $2^{\text {nd }}-3^{\text {rd }}$ trimesters of gestation in the peripheral blood of pregnant women with bacterial and viral infections of the lower portion of the urogenital tract.

\section{Materials and methods}

The study implied an assessment of surface phenotype of monocytes in the $2^{\text {nd }}$ and $3^{\text {rd }}$ trimesters in the pregnant with bacterial and viral urogenital infections (UGI). The control group involved patients with physiological pregnancy. UGI diagnosis was based on a comprehensive assessment of clinical presentation and laboratory findings. Infectious status of patients was determined using bacteriological method, polymerase chain reaction (PCR), and enzyme immunoassay (EIA). Identification of Ig A and Ig G to Cl. Trachomatis and chlamydial antigen was considered to be the basis for diagnosis of chronic chlamydial infection. Persistent herpes virus infection was diagnosed following the detection of specific antibodies to HSV (I, II, VI), and HSV DNA detection by PCR. Expression of surface markers of monocyte/macrophage cells in the peripheral venous blood of the pregnant was assessed by flow cytophluorometry using sets produced by Becton Dickinson, USA on flow cytometer facscan by standard methods. We used FITC labeled monoclonal antibodies, particularly anti-CD16, anti-CD25, anti-CD95 and phycoerythrinlabeled anti-HLA-DR. Statistical data processing was carried out using a general-purpose processing software Statistica for Windows 6.1 (Russian version).

\section{Main results}

In the second trimester the study group was found to have a reduction in CD16 + (p <0.01), CD25 + (p <0.02), CD95 + (p $<0.05$ ), and HLA-DR-monocytes ( $p<0.05$ ) compared with the control group. CD95+ molecule is an apoptosis inducer which means that its expression reduction on the surface of monocytes in the second trimester of gestation may lead to inhibition of apoptosis and disruption of adequate implantation due to lack of dilatation of placental vessels, which results in deterioration of placental circulation. CD16 +, being a third type Fc-receptor component, mediates antibody-dependent cytotoxicity (ADCT) reaction, which can inhibit the function of foreign component or infectious agent destruction in the pregnant. In the $3^{\text {rd }}$ trimester the patients were found to have an increase in CD16 +, CD95 + , HLA-DR + monocytes and a significant decrease in CD25+monocytes in pregnancies complicated by UGI. The increase in CD95 + expression indicates an increase in apoptosis, which can lead to impaired blood flow at the local level and contribute to the "aging" of the placenta.

\section{Conclusion}

Increased expression of HLA-DR molecules on monocytes in the $3^{\text {rd }}$ trimester may indicate hyper-reactivity of the body in the late period of gestation, enhancing cytotoxic potential of the immune system, which can apparently cause morphological changes in the placenta and, accordingly, its function disorders. 